\title{
Skin Hydration Disrupts the Stratum Corneum Barrier Lipids with Formation of Large Internal Water Pockets
}

\author{
Ronald R. Warner*, Keith J. Stone** and Ying L. Boissy* \\ *Miami Valley Laboratories, Procter \& Gamble Co., Cincinnati, Ohio 45253 \\ **Winton Hill Technical Center, Procter \& Gamble Co., Cincinnati, Ohio 45224
}

It is increasingly apparent that prolonged water contact is deleterious to skin. Water exposure has long been known to increase skin permeability [1], and prolonged water contact is an important factor in the causation of irritant contact dermatitis [2]. The mechanism by which water increases permeability and promotes dermatitis is unknown. However, since the primary permeability barrier of the skin is the structured lamellar lipids of the stratum corneum (SC) [3], it is reasonable to suspect that prolonged water exposure may be disrupting lipid order.

Previously we showed by transmission electron microscopy (TEM) that prolonged exposure of porcine skin to warm $\left(46^{\circ} \mathrm{C}\right)$ water in vitro did cause extensive disruption of the SC lipid ultrastructure [4]. Alterations in intercellular lipid structure were detected after 2 hours, and nearly complete delamination and corneocyte separation were observed after 24 hours of water exposure. Similar but less severe effects were observed in human skin in vivo after 24 hours of water exposure.

In this study we confirm that extended water exposure disrupts human SC intercellular lipid lamellae in vivo. Untreated and water exposed regions of the same forearm were biopsied and the biopsy cut in half, one piece being placed in fixative for TEM, the other plunged into cooled liquid ethane for cryo-SEM. As shown in the TEM images of Figure 1, water exposure leads to disruption of the SC intercellular lipid lamellae with formation of clear regions that presumably contained water prior to plastic embedding. By cryo-SEM, water exposure for 4 or 24 hours resulted in, respectively, a 3 or 4-fold expansion of the SC thickness. As shown in Figure 2, with water exposure large pockets of water ("cisternae") form within the intercellular lamellar lipid space. Cisternae increase in size and number with time of water exposure. Within cisternae the lipid structure is disrupted by lamellar delamination. Cisternae appear tobe disk-shaped structures that do not obviously communicate. They appear to contain considerable lipid-like material, to communicate with salts in the external solution, and to contain a substantial fluid volume rivaling the volume of the dry SC. We suggest that the SC is a more chaotic structure than previously envisioned. This work provides a framework for better understanding skin irritancy and percutaneous transport.

\section{References}

[1] R. Scheuplein, The Physiology and Pathophysiology of the Skin (A. Jarrett, ed.), Academic Press, London, 1978, pp 1731-1752.

[2] L. Halkier-Sørensen, et al., The Irritant Contact Dermatitis Syndrome ( P.G.M. van der Valk \& H.I. Maibach, eds.), CRC Press, Boca Raton, Florida, 1995, pp 23-52.

[3] P.M. Elias, Arch Dermatol Res 270 (1981) 95-117.

[4] R.R. Warner, et al., J Invest Dermatol 113 (1999) 960-966. 


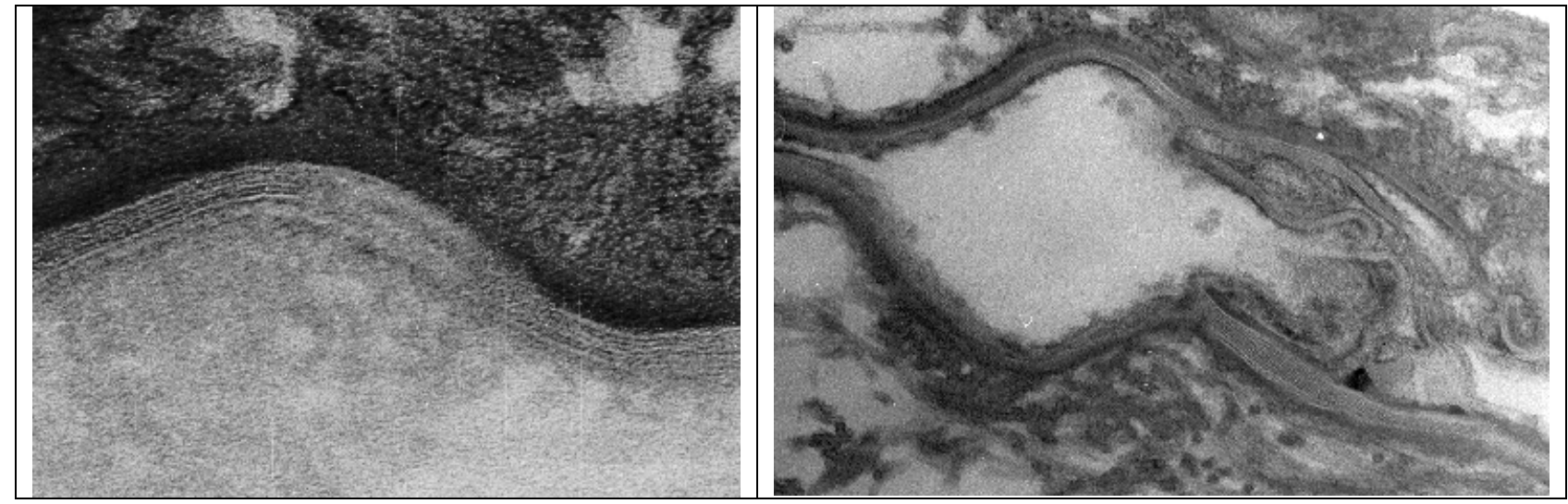

Figure 1. Left image, lamellar lipids of the control skin fill the SC intercellular space. On the right, following $24 \mathrm{hr}$ water treatment, the intercellular space is widened and lipids disrupted. 160,000x.

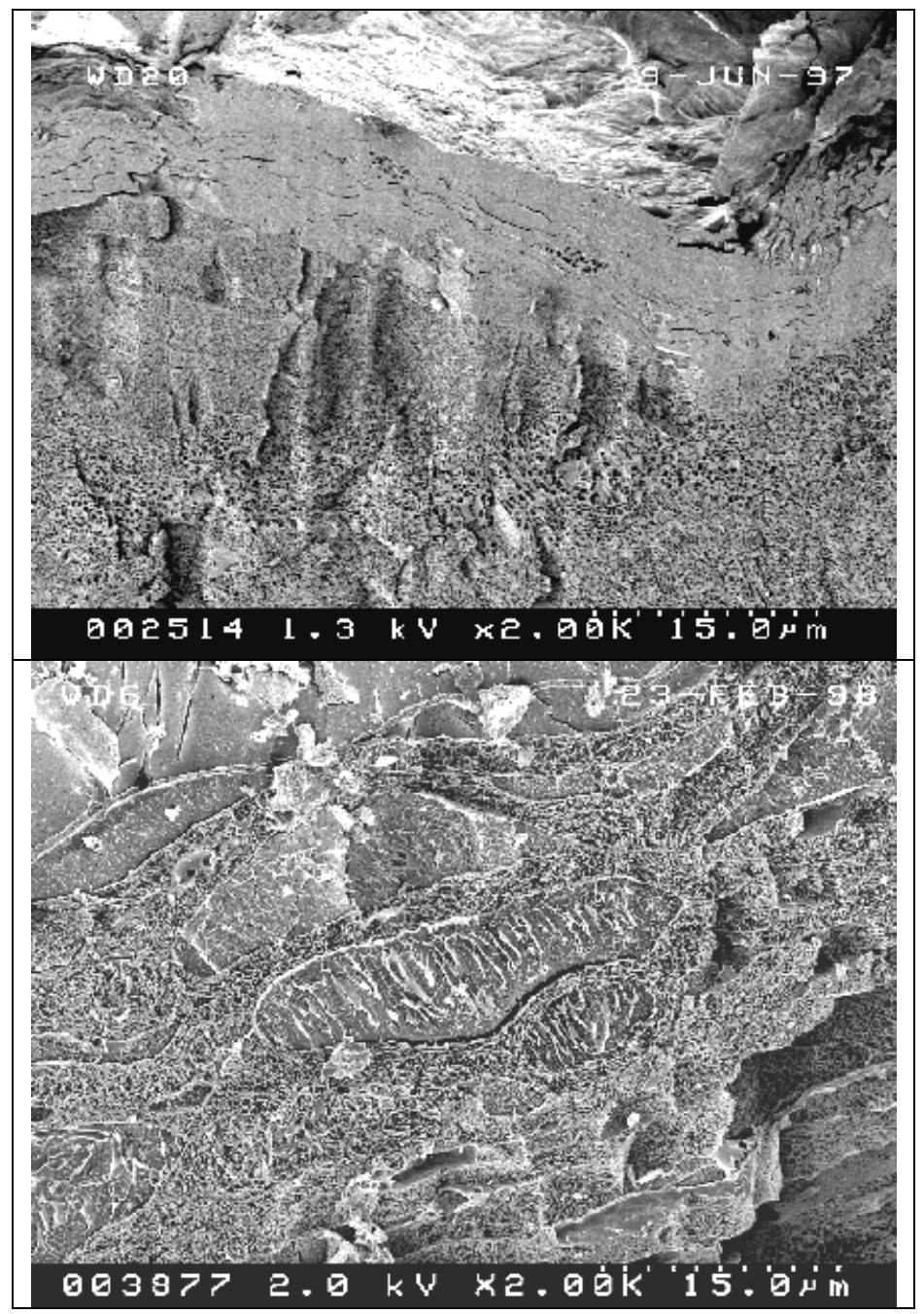

Figure 2. Top: Cryo-SEM of untreated skin showing a 10um thick SC. Bottom: After $24 \mathrm{hr}$ water exposure, the SC swells to $40 \mu \mathrm{m}$ and large pools of fluid are present in the intercellular space. 